\title{
Temperature and light-induced self-assembly changes of a tetra-arm diblock copolymer in an ionic liquid
}

\author{
Xiaofeng Ma, Ryoji Usui, Yuzo Kitazawa, Hisashi Kokubo and Masayoshi Watanabe \\ A tetra-arm diblock copolymer ([PEG- $b$-P(AzoMA-r-NIPAm) $]_{4}$ ) was synthesized by reversible addition-fragmentation chain transfer \\ copolymerization of $\mathrm{N}$-isopropylacrylamide (NIPAm) and 4-phenylazophenyl methacrylate (AzoMA), initiating from the ends of \\ functionalized tetra-arm polyethylene glycol (tetra-PEG). The resulting tetra-arm diblock copolymer consists of two segments: \\ tetra-PEG (the center block; compatible with 1-butyl-3-methylimidazolium hexafluorophosphate $\left(\left[\mathrm{C}_{4} \mathrm{mim}_{\mathrm{P}} \mathrm{PF}_{6}\right)\right.$ ) and $\mathrm{P}(\mathrm{Azo} \mathrm{MA}-\mathrm{r}$ - \\ NIPAm) (the end blocks; temperature- and photosensitive compatibility with $\left[\mathrm{C}_{4} \mathrm{mim}_{\mathrm{PFF}}\right.$ due to photoresponsive upper critical \\ solution temperature phase behavior). We found that $[\mathrm{PEG}-b \text {-P(AzoMA-r-NIPAm) }]_{4}$ underwent high-temperature unimer and low- \\ temperature micelle (upper critical micellization temperature (UCMT)) transitions in $\left[\mathrm{C}_{4} \mathrm{mim}_{\mathrm{P}} \mathrm{PF}_{6}\right.$. The UCMT of the tetra-arm \\ diblock copolymer depended on photoisomerization states of the azobenzene groups within the copolymer. The UCMT of the \\ trans-form polymer in dark conditions was higher than that of the cis-form polymer under UV-light irradiation. We demonstrated \\ photoinduced self-assembly changes of the tetra-arm diblock copolymer in $\left[\mathrm{C}_{4}\right.$ mim]PF 6 at a 'bistable' temperature. Reversible \\ photoinduced unimer/micelle transitions were also demonstrated.
}

Polymer Journal (2015) 47, 739-746; doi:10.1038/pj.2015.55; published online 29 July 2015

\section{INTRODUCTION}

Ionic liquids (ILs) are ambient temperature molten salts and have attracted considerable attention because of their unique properties such as nonvolatility, nonflammability, thermal and chemical stability and high ionic conductivity. ${ }^{1-8}$ Composite materials consisting of polymers and ILs can realize useful materials such as polymer electrolyte membranes, gas separation membranes and catalytic membranes. ${ }^{9,10}$ Furthermore, we previously focused on stimuliresponsive polymers in ILs. ${ }^{11,12}$ For example, poly( $N$-isopropylacrylamide) (PNIPAm) shows an upper critical solution temperature (UCST) phase transition in certain ILs, which is a completely opposite phenomenon to that observed in aqueous solutions. ${ }^{13}$ In contrast, certain polymethacrylates ${ }^{14-16}$ or polyether derivatives ${ }^{17-19}$ are found to exhibit lower critical solution temperature (LCST) phase behavior in ILs. Phase transitions of polymers in nonvolatile and thermally stable ILs can afford long-term stable smart materials without solvent evaporation. Recently, we developed photoresponsive polymers in ILs by copolymerization of an azobenzene-containing monomer, 4-phenylazophenyl methacrylate (AzoMA), with either $\mathrm{NIPAm}^{20}$ or benzyl methacrylate ${ }^{21}$ as the main monomer.

Self-assembly of block copolymer in an IL is another fascinating topic from the materials science point of view. ${ }^{22-26}$ Block copolymer membranes or dilute solutions exhibit useful nanostructures due to intramolecular segregation. Lodge and coworkers reported polybutadiene-block-poly(ethylene oxide) (PB-b-PEO) block copolymer selfassembly in 1-butyl-3-methylimidazolium hexafluorophosphate
$\left(\left[\mathrm{C}_{4} \mathrm{mim}\right] \mathrm{PF}_{6}\right) .{ }^{27}$ They found that PB- $b$-PEO self-assembled into micelles with an IL-insoluble PB core surrounded by PEO corona chains in $\left[\mathrm{C}_{4} \mathrm{mim}\right] \mathrm{PF}_{6}$. Direct visualization using cryogenic transmission electron microscopy revealed various nano-ordered selfassembled structures with universal morphologies such as spheres, cylindrical micelles and bilayer vesicles in $\left[\mathrm{C}_{4} \mathrm{mim}\right] \mathrm{PF}_{6}$. They also developed physical ion gels by gelation of an ABA triblock copolymer with an IL-selective B block and an IL-phobic A block in an IL. For example, polystyrene-block-poly(ethylene oxide)-block-polystyrene triblock copolymer dissolved in a PEO selective IL can form an ion gel through noncovalent association of the polystyrene blocks. ${ }^{28}$ Physical ion gels comprising ABA triblock copolymers and ILs have numerous applications including organic transistors ${ }^{29}$, actuators ${ }^{30}$ and lowvoltage, flexible electrochemiluminescent device. ${ }^{31}$ Certain thermosensitive block copolymers in ILs can realize thermoinduced micellization, ${ }^{32}$ doubly thermosensitive micelles, ${ }^{33,34}$ and thermoreversible ion gels. ${ }^{35-37}$ We also reported that an azobenzene-containing diblock copolymer (PEO- $b$-P(AzoMA- $r$-NIPAm)) exhibits thermoand photoinduced micellization in $\left[\mathrm{C}_{4} \mathrm{mim}\right] \mathrm{PF}_{6} \cdot{ }^{38}$ Owing to the UCST phase behavior of random copolymer segments composed of AzoMA and NIPAm (P(AzoMA-r-NIPAm)), the diblock copolymer formed micelles at lower temperatures than the UCST of P(AzoMA-rNIPAm). $\left[\mathrm{C}_{4} \mathrm{mim}\right] \mathrm{PF}_{6}$ was selected because the UCST locates at experimentally suitable temperatures. Conversely, the block copolymer became unimers at higher temperatures than the UCST. The upper critical micellization temperature (UCMT) depended on the 
photoisomerization states of azobenzene in the copolymer. The UCMT difference between the trans-form polymer and the cis-form polymer was $4^{\circ} \mathrm{C}$. At a suitable temperature, photoinduced reversible unimer/micelle transition was realized by illumination using light with appropriate wavelength. Very recently, we succeeded in realizing the sol-gel transition of concentrated ABA triblock copolymer solutions (20 wt \%) in an IL triggered by a photostimulus. ${ }^{39}$ The ABA triblock copolymer employed was P(AzoMA- $r$-NIPAm)- $b$-PEO- $b$-P(AzoMA- $r$ NIPAm), and the sol-gel transition was caused by the photoinduced aggregation state change of the ABA triblock copolymer. The photo processable ion gel is a very fascinating material; however, mechanical strength of the ion gel was not sufficient. ${ }^{39}$

How block copolymer architecture affects the aggregation states is of great interest. We need to accumulate such knowledge because the information on self-assembly of block copolymer in ILs is rather poor, compared with that in water and organic solvents. Furthermore, in order to utilize the photoinduced sol-gel transition for material processing, the enhancement of mechanical integrity of the gel state is our great concern. Herein, we describe thermo- and photoinduced self-assembly changes of a tetra-arm diblock copolymer ([PEG- $b$-P (AzoMA- $r$-NIPAm) $]_{4}$ ) in $\left[\mathrm{C}_{4} \mathrm{mim} \mathrm{PF}_{6}\right.$. The [PEG- $b-\mathrm{P}($ AzoMA- $r-$ $\mathrm{NIPAm})]_{4}$ diblock copolymer, with tetra-polyethylene glycol (tetraPEG) as the central block and P(AzoMA-r-NIPAm) as the four end blocks, was prepared by reversible addition-fragmentation chain transfer (RAFT) copolymerization of NIPAm and AzoMA, initiating from end functionalized tetra-PEG precursor. Our paper first describes the thermosensitive self-assembly of the tetra-arm diblock copolymer in $\left[\mathrm{C}_{4} \mathrm{mim}\right] \mathrm{PF}_{6}$. Then it describes the reversible photoinduced unimer/micelle transitions by alternating irradiation by UV and visible light at a suitable temperature. Although micellization of tetra-arm diblock copolymers can be found in aqueous solutions, ${ }^{40-47}$ selfassembly of tetra-arm diblock copolymers in ILs are yet to be reported.

\section{EXPERIMENTAL PROCEDURE}

\section{Materials}

Tetra-PEG (number-average molecular weight $\left(M_{\mathrm{n}}\right)=40000$, average molecular weight of each arm $=10000$; polydispersity index $\left.\left(M_{\mathrm{w}} / M_{\mathrm{n}}\right)=1.05\right)$ was provided by NOF Corporation (Tokyo, Japan) and dried by azeotropic distillation using toluene to remove residual water. NIPAm was provided by Kohjin Chemicals Corporation (Tokyo, Japan) and purified by recrystallization (two times) using a toluene/hexane (1:10 by weight) mixed solvent. 2,2'-Azobis (isobutyronitrile) was purchased from Sigma-Aldrich (Tokyo, Japan) and recrystallized from methanol. 1,4-Dioxane, dehydrated dichloromethane and oxalyl chloride were purchased from Wako Chemicals (Osaka, Japan) and used without further purification. $S$-1-dodecyl- $S^{\prime}-\left(\alpha, \alpha^{\prime}\right.$-dimethyl- $\alpha^{\prime \prime}$-acetic acid $)$ trithiocarbonate (CTA) was synthesized according to a previously reported procedure. ${ }^{48,49}$ AzoMA was synthesized by a reported procedure. ${ }^{50,51}\left[\mathrm{C}_{4} \mathrm{mim}\right]$ $\mathrm{PF}_{6}$ was prepared according to a previous report. ${ }^{52}$ The water content of the $\left[\mathrm{C}_{4} \mathrm{mim}\right] \mathrm{PF}_{6}$ was determined by a Karl Fischer titration and was <10 p.p.m.

\section{Synthesis of [PEG- $b$-P(AzoMA-r-NIPAm) $]_{4}$ tetra-arm diblock copolymer}

The block copolymer was prepared according to the procedure shown in Scheme 1. The first step was to synthesize the tetra-PEG-CTA macroinitiator Tetra-PEG $(6.64 \mathrm{~g}, 0.166 \mathrm{mmol})$ and dry dichloromethane $(50 \mathrm{ml})$ was placed into a two-necked flask and stirred at room temperature until a transparent solution was obtained. CTA $(0.620 \mathrm{~g}, 1.70 \mathrm{mmol})$ was mixed with excess oxalyl chloride $(0.44 \mathrm{ml}, 5.1 \mathrm{mmol})$ in dry dichloromethane $(10 \mathrm{ml})$ in another twonecked flask under an argon atmosphere and stirred at room temperature until gas evolution stopped $(\sim 2 \mathrm{~h})$. Dichloromethane and excess oxalyl chloride were then removed under vacuum, and the residue was re-dissolved in dry dichloromethane $(12 \mathrm{ml})$. This solution was then added to tetra-PEG solution. The reaction was allowed to stir for $24 \mathrm{~h}$ at room temperature, after which the contents were reprecipitated three times by pouring the tetra-PEG-CTA solution in tetrahydrofuran (as a good solvent) into cold diethyl ether (as a poor solvent). The precipitate was then dried under vacuum at room temperature overnight to yield tetra-PEG-CTA as a light-yellow powder (6.33 g, 90\%).

The second step was RAFT copolymerization of NIPAm and AzoMA from the end of the obtained tetra-PEG-CTA. Tetra-PEG-CTA (1.00 g, 0.0242 $\mathrm{mmol})$, AzoMA $(1.36 \mathrm{~g}, 5.11 \mathrm{mmol})$ and $\mathrm{NIPAm}(15.9 \mathrm{~g}, \quad 0.141 \mathrm{~mol})$ $([$ AzoMA $] /[$ NIPAm $]=3.5 / 96.5)$ were dissolved in 1,4 -dioxane $(100 \mathrm{ml})$ in a round-bottom flask and bubbled with argon for $30 \mathrm{~min}$ at $45^{\circ} \mathrm{C}$. Special attention was given to avoid crystallization of the NIPAm monomer during the bubbling process. 2,2'-Azobis(isobutyronitrile) $(5.0 \mathrm{mg}, 0.030 \mathrm{mmol})$ and 1,4-dioxane $(5.0 \mathrm{ml})$ was separately placed in a separate round-bottom flask and bubbled with argon for $30 \mathrm{~min}$ at room temperature. The 2,2'Azobis (isobutyronitrile) solution $(3.3 \mathrm{ml}, 0.020 \mathrm{mmol})$ was then added to the monomer solution. RAFT polymerization was carried out at $65^{\circ} \mathrm{C}$ for $68 \mathrm{~h}$. The reaction mixture was evaporated and purified by reprecipitation three times from acetone (as a good solvent) to cold diethyl ether (as a poor solvent). Then, the collected yellow powder was purified by fractional precipitation. The precipitate was dissolved in ethanol with $5 \mathrm{wt} \%$ concentration at $40^{\circ} \mathrm{C}$. After the solution was stored in a refrigerator about 2 days, the solid precipitate was observed. Then the upper solution was decanted and the precipitate was collected. The precipitate was purified again by reprecipitation with acetone and cold diethyl ether. The precipitate was then dried overnight under vacuum at $40{ }^{\circ} \mathrm{C}$ to yield [PEG- $b$-P(AzoMA- $r$-NIPAm)-CTA $]_{4}$ as a yellow powder $(2.10 \mathrm{~g}, 11 \%)$.

Finally, the removal of the RAFT agent at each chain end of the polymer ([PEG- $b$-P(AzoMA- $r$-NIPAm)-CTA $]_{4}$ ) was conducted according to the following procedure. [PEG- $b-\mathrm{P}(\text { AzoMA- } r \text {-NIPAm)-CTA }]_{4}(1.00 \mathrm{~g}, 0.0107 \mathrm{mmol})$, 2,2'-Azobis(isobutyronitrile) $(0.212 \mathrm{~g}, 1.29 \mathrm{mmol})$ and 1,4 -dioxane $(25 \mathrm{ml})$ were mixed in a round-bottom flask and bubbled with argon for $30 \mathrm{~min}$ at room temperature. The reaction was allowed to proceed under argon atmosphere at $80^{\circ} \mathrm{C}$ for $12 \mathrm{~h}$. The reaction mixture was evaporated and purified by reprecipitation three times from acetone (as a good solvent) to cold diethyl ether (as a poor solvent). The precipitate was then dried overnight under vacuum at $40{ }^{\circ} \mathrm{C}$ to yield [PEG- $b$-P(AzoMA- $r$-NIPAm $\left.)\right]_{4}$ as a yellow powder $(0.870 \mathrm{~g}, 87 \%)$.

\section{Sample preparation}

A solution of $[\mathrm{PEG}-b-\mathrm{P}(\mathrm{AzoMA}-\mathrm{r}-\mathrm{NIPAm})]_{4}$ in $\left[\mathrm{C}_{4} \mathrm{mim}\right] \mathrm{PF}_{6}$ was prepared using the co-solvent method. The tetra-arm diblock copolymer was first dissolved in tetrahydrofuran, followed by the addition of an appropriate amount of $\left[\mathrm{C}_{4} \mathrm{mim}\right] \mathrm{PF}_{6}$ (polymer/IL $=1 / 99$ by weight). The solution was then stirred continuously for at least $3 \mathrm{~h}$ until a transparent solution was obtained. The volatile tetrahydrofuran was then removed by evaporation at $80^{\circ} \mathrm{C}$ under vacuum for $24 \mathrm{~h}$. Sample solution was filtered by using $0.20-\mu \mathrm{m}$ filter to eliminate dust before dynamic light scattering (DLS) measurements.

\section{Measurements}

The synthesized polymers were characterized by ${ }^{1} \mathrm{H}$ NMR and gel permeation chromatography (GPC). ${ }^{1} \mathrm{H}$ NMR $(500 \mathrm{MHz})$ spectrum was recorded with a Bruker DXR 500 (Karlsruhe, Germany). The $M_{\mathrm{w}} / M_{\mathrm{n}}$ of the synthesized polymers was determined by GPC using a Shimadzu HPLC system (columns: Tosoh calibrated by polystyrene standards (Kyoto, Japan)). The system was operated at a flow rate of $1.0 \mathrm{ml} \mathrm{min}{ }^{-1}$ using dimethylformamide containing $0.01 \mathrm{moll}^{-1} \mathrm{LiBr}$ as the eluent.

DLS was performed using an Otsuka Electronics DLS-6500 (Osaka, Japan) equipped with an ALV-6010/160 correlator (Langen, Germany) and a He-Ne laser with wavelength of $633 \mathrm{~nm}$. Temperatures were controlled to within an accuracy of $\pm 0.1^{\circ} \mathrm{C}$ using an index-matching silicon oil bath. Experiments were performed at various temperatures from 75 to $50^{\circ} \mathrm{C}$ in a cooling process. The intensity correlation functions $g_{2}(q, t)$ were recorded at a scattering angle of $90^{\circ}$ at each temperature for $30 \mathrm{~s}$ to obtain the relationship between $R_{\mathrm{h}}$ values and temperatures. The intensity correlation function data for photoreversible micellization were collected every $30 \mathrm{~s}$ to make the time resolution of each measurement, which is faster than that of the photoinduced micellization 


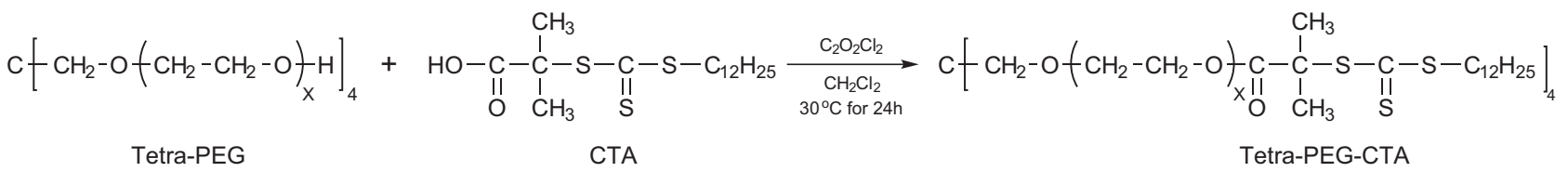
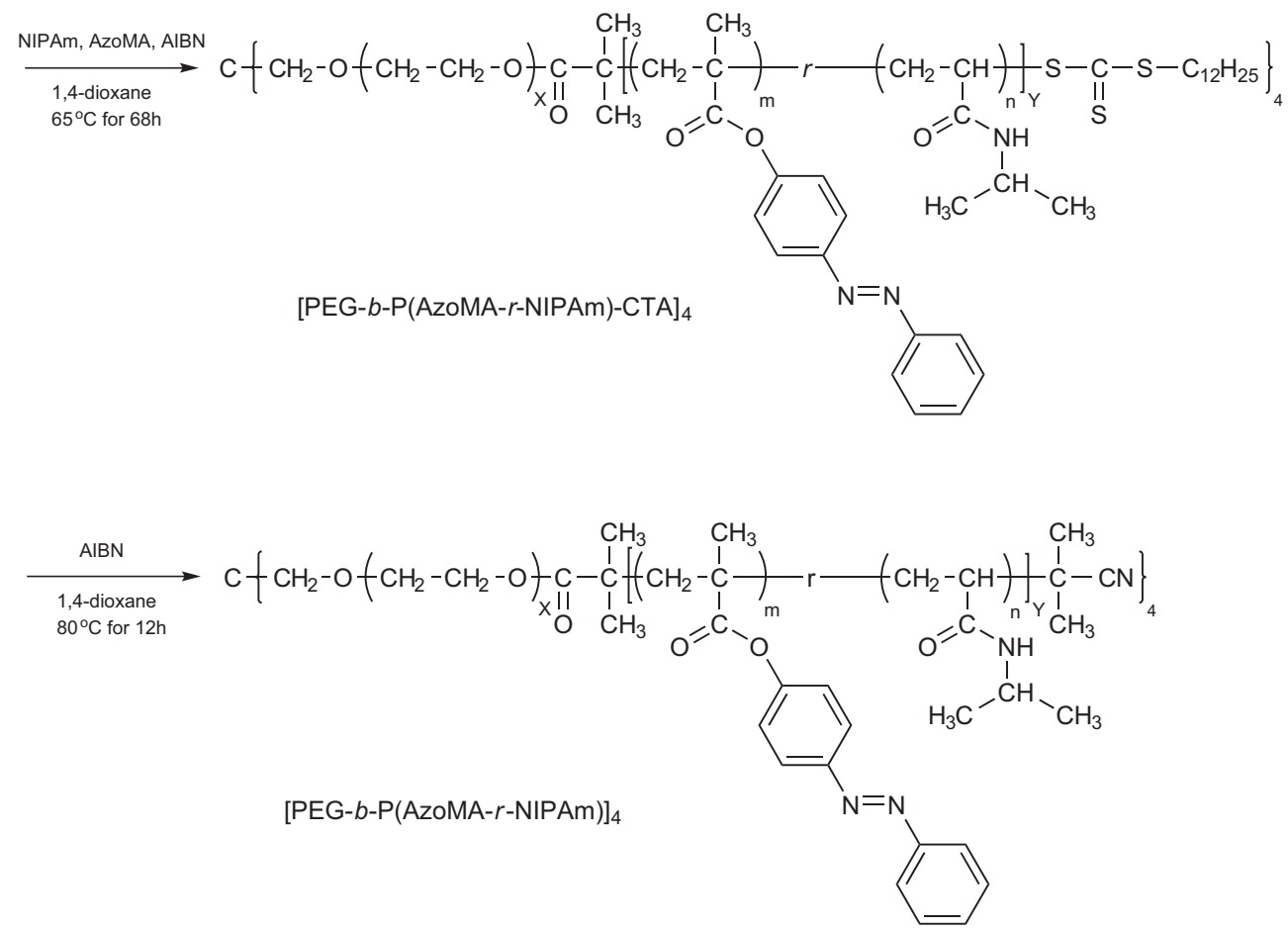

Scheme 1 Synthetic procedure for the tetra-arm diblock copolymer.

process. Data was collected after equilibrating at each temperature for at least $30 \mathrm{~min}$.

For solutions containing monodisperse particles, the electric field correlation function $g_{1}(q, t)$ exhibits a single exponential decay,

$$
g_{1}(q, t)=\exp (-\Gamma t)=\exp \left(-D_{0} q^{2} t\right)
$$

where $t$ is the time, $q$ is the scattering vector $(q=(4 \pi n / \lambda) \sin (\theta / 2) ; n$ is the refractive index of the solutions, $\lambda$ is the wavelength of the light in vacuum and $\theta$ is the scattering angle), $\Gamma$ is the decay rate and $D_{0}$ is the mutual diffusion coefficient at infinite dilution. The recorded intensity correlation function $g_{2}(q, t)$ was converted to $g_{1}(q, t)$ through the Siegert relation..$^{53}$ The hydrodynamic radius, $R_{\mathrm{h}}$, can be estimated using the solvent viscosity, $\eta$ and the Stokes-Einstein equation,

$$
R_{\mathrm{h}}=\left(k_{\mathrm{b}} T\right) / 6 \pi \eta D_{0}
$$

where $k_{\mathrm{b}}$ and $T$ are the Boltzmann constant and absolute temperature, respectively. For solutions with polydisperse (not monomodal) particles, $g_{1}(q$, t) can be analyzed by the method of cumulants, ${ }^{54}$

$$
g_{1}(q, t)=A \exp (-\Gamma t)\left(1+(1 / 2 !) \mu_{2} t^{2}-(1 / 3 !) \mu_{3} t^{3}\right),
$$

where $\Gamma$ is the mean decay rate and $\mu_{2} / \Gamma^{2}$ represents the width of the distribution. In this work, the apparent hydrodynamic radius, $R_{\mathrm{h}}$, was determined using equation (2) by replacing $D_{0}$ with $D=\Gamma / q^{2}$ of the $1 \mathrm{wt} \%$ solutions. The distribution of $R_{\mathrm{h}}$ was also obtained by applying the inverse Laplace transformation to $g_{1}(q, t)$ with the well-established CONTIN program, ${ }^{55}$ and by a sum of two exponentials. To estimate $R_{\mathrm{h}}$, the temperature dependence of the viscosity of $\left[\mathrm{C}_{4} \mathrm{mim}\right] \mathrm{PF}_{6}$ was calculated using the appropriate Vogel-Tammann-Fulcher equation, ${ }^{52}$

$$
\eta=0.36 \exp [639 /(T-201)]
$$

where $T(\mathrm{~K})$ is the absolute temperature. The refractive index of $\left[\mathrm{C}_{4} \mathrm{mim}\right] \mathrm{PF}_{6}$ was calculated using equation, ${ }^{56}$

$$
n=1.49569-3.2 \times 10^{-4} \mathrm{~T}+8.8 \times 10^{-8} T^{2}
$$

where $T(\mathrm{~K})$ is the absolute temperature. Photoirradiation was carried out using a $500 \mathrm{~W}$ high-pressure mercury lamp (Ushio Optical Modulex BA-H500). The wavelength and intensity of the irradiated light (UV-light: $366 \mathrm{~nm}$, $8 \mathrm{~mW} \mathrm{~cm}^{-2}$; visible light: $437 \mathrm{~nm}, 4 \mathrm{~mW} \mathrm{~cm}^{-2}$ ) were adjusted using color filters. Either UV $(366 \mathrm{~nm})$ or visible light $(437 \mathrm{~nm})$ was irradiated from the upper side of the DLS sample tube $(1 \mathrm{~cm}$ diameter). A transparent heatabsorbing filter covered the top of the sample tube to avoid the generation of heat from the mercury lamp and contamination by dust.

\section{RESULTS AND DISCUSSION}

Characterization of the tetra-arm diblock copolymer

The products of each reaction step in Scheme 1 were identified by ${ }^{1} \mathrm{H}$ NMR (Supplementary Figures S-1, and Figure 1). The end group conversion ratio of tetra-PEG-CTA was calculated from the ${ }^{1} \mathrm{H}$ NMR spectrum (Supplementary Figure S-1) by comparing integrated signal intensities of the ethylene protons in the tetra-PEG main chain (e) with that of the methyl protons in CTA (a) and was found to be unity. Changes in the GPC traces with each reaction step are shown in Figure 2, and an ${ }^{1} \mathrm{H}$ NMR spectrum of the target block copolymer 


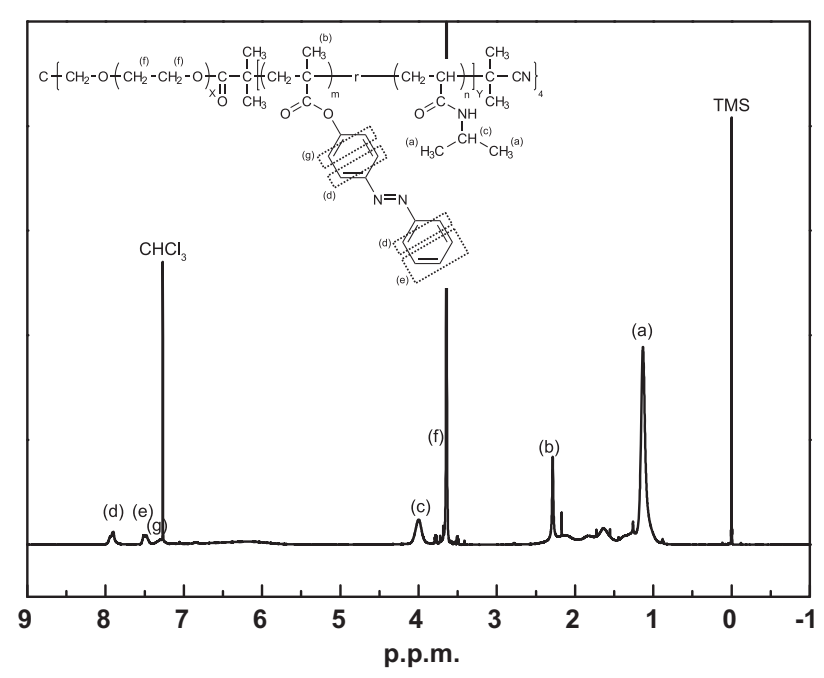

Figure $1{ }^{1} \mathrm{H}$ NMR spectrum of [PEG- $b$-P(AzoMA-r-NIPAm) $]_{4}$ in $\mathrm{CDCl}_{3}$.

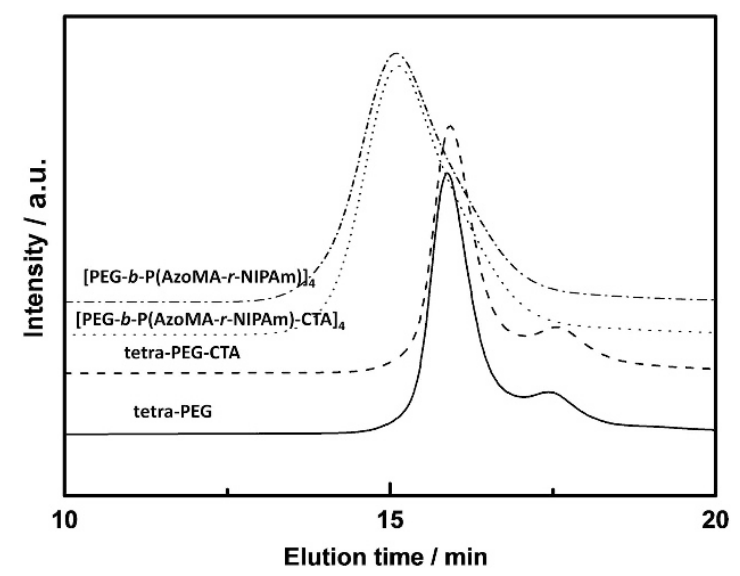

Figure 2 GPC traces of tetra-PEG (solid line), tetra-PEG-CTA (dashed line), $\left[\right.$ PEG- $b-P(\text { AzoMA-r-NIPAm)-CTA }]_{4}$ (dotted line) and [PEG- $b-P(A z o M A-r-$ $\mathrm{NIPAm})]_{4}$ (dashed and dotted line).

([PEG- $b$-P(AzoMA- $b$-NIPAm $\left.)]_{4}\right)$ is shown in Figure 1 . The $M_{\mathrm{n}}$ of $[\text { PEG- } b \text {-P(AzoMA- } b \text {-NIPAm) }]_{4}$ was calculated from the ${ }^{1} \mathrm{H}$ NMR spectrum (Figure 1) by comparing the integrated signal intensities of the tetra-PEG main chain ((f) 3.6 p.p.m.) with that of the end blocks ((c) 4.0 p.p.m. from NIPAm; (d) 7.9 p.p.m. from AzoMA). The AzoMA composition was calculated from the ratio of integrated signal intensities between NIPAm and AzoMA, which showed that $8.7 \mathrm{~mol} \%$ of AzoMA was incorporated into the random copolymer. The characterized results of the original tetra-PEG and the synthesized tetra-arm polymers are summarized in Table 1.

In the GPC trace of tetra-PEG, a satellite peak was observed at a longer elution time than that of the main peak, which corresponded to PEG having $M_{\mathrm{n}}=10 \mathrm{kDa}$, one-fourth of $M_{\mathrm{n}}$ of tetra-PEG, which appeared to be originated from trace amount of water in the preparation system. This satellite peak still persisted in the GPC trace of tetra-PEG-CTA. In contrast, the GPC traces of [PEG- $b$-P(AzoMA- $r$ NIPAm $)-C T A]_{4}$ and $[$ PEG- $b$-P(AzoMA- $r$-NIPAm $\left.)\right]_{4}$ were unimodal, indicating that low molecular weight block copolymers starting from the low molecular weight PEG were effectively removed by the fractional precipitation (Supplementary Figure S-3). Although
Table 1 Molecular characterization of tetra-PEG and tetra-arm block copolymer ([PEG-b-P(AzoMA-r-NIPAm) $]_{4}$ )

\begin{tabular}{llcc}
\hline Polymer & $M_{\mathrm{n}}(\mathrm{kDa})$ & $M_{\mathrm{w}} / M_{\mathrm{n}}{ }^{\mathrm{a}}$ & [AzoMA]/[NIPAm ${ }^{\mathrm{P}}$ \\
\hline Tetra-PEG & 40.0 & 1.05 & - \\
{$[\text { PEG- } b \text {-P(AzoMA-r-NIPAm) }]_{4}$} & $92.0^{\mathrm{b}}$ & 1.41 & $8.7 / 91.3$ \\
\hline
\end{tabular}

Abbreviations: AzoMA, 4-phenylazophenyl methacrylate; DMF, dimethylformamide; GPC, gel permeation chromatography; NIPAm, N-isopropylacrylamide; PEG, polyethylene glycol; PSt, polystyrene.

aDetermined by GPC (calibrated with PSt standards and eluent: DMF containing

$0.01 \mathrm{moll}^{-1} \mathrm{LiBr}$.

${ }^{\mathrm{b}}$ Determined by ${ }^{1} \mathrm{HNMR}$.

polymers prepared by living radical polymerization usually have a lower $M_{\mathrm{w}} / M_{\mathrm{n}}$ than 1.2. However, [PEG- $b-\mathrm{P}(\text { AzoMA- } r \text {-NIPAm) }]_{4}$ has $M_{\mathrm{w}} / M_{\mathrm{n}}=1.4$. This is due to the fact that the polymerization is less controlled due to retarding or inhibiting effect of the azobenzene group for radicals. ${ }^{57}$ All of the data supported reasonable preparation of the tetra-arm diblock copolymer ([PEG- $b$-P(AzoMA- $r$-NIPAm $\left.)]_{4}\right)$.

\section{Thermosensitivity of the tetra-arm diblock copolymer in $\left[\mathrm{C}_{4} \mathrm{mim}\right] \mathrm{PF}_{6}$}

DLS was employed to investigate micellization of tetra-arm diblock copolymer in $\left[\mathrm{C}_{4} \mathrm{mim}\right] \mathrm{PF}_{6}$. Figure 3 shows the normalized scattering intensity and hydrodynamic radius of a $1 \mathrm{wt} \%$ tetra-arm diblock copolymer solution in $\left[\mathrm{C}_{4} \mathrm{mim}\right] \mathrm{PF}_{6}$ with or without UV-light irradiation at a scattering angle of $90^{\circ}$. The normalized scattering intensities were defined as the intensity at each temperature divided by that at $75^{\circ} \mathrm{C}$. Under dark conditions, the isomerization state of the azobenzene was composed of the trans-state $(100 \%)$, whereas under UV-light irradiation the cis-state $(80 \%)$ dominated..$^{21,58}$ In the cooling process from $75^{\circ} \mathrm{C}$, the scattered intensity increased with decrease of sample temperature in the dark and under UV-light irradiation (Figure 3a), thanks to the UCST phase behavior of the P(AzoMA-r-NIPAm) segments. This indicates that the tetra-arm diblock copolymer aggregates into large size micelles, which scatter the incident light. The aggregation behavior was thermally reversible. The mean hydrodynamic radius $\left(R_{\mathrm{h}}\right)$ of the trans- and cis-form polymer were also determined (Figure $3 \mathrm{~b}$ ). At higher temperatures than the UCMT, the distribution function was bimodal both in the dark and under UV-light irradiation (Figures 4 and 5). The smaller $R_{\mathrm{h}}$ peak corresponds to the single polymer chains (unimers), whereas the larger $R_{\mathrm{h}}$ peak indicates the existence of aggregates. (dashed lines in Figure $3 \mathrm{~b}$ ) However, the weight fraction of the unimers is calculated to be over 0.999 using a previously reported method, ${ }^{59}$ and it was considered that almost all of the scattering in $\left[\mathrm{C}_{4} \mathrm{mim}\right] \mathrm{PF}_{6}$ above the UCMT came from single polymer chains. There are two possible reasons for this small amount of aggregates at higher temperatures. One reason is that these aggregates might arise from solubility differences because of chain-to-chain variations in the comonomer distribution and composition in the random copolymer block; the other reason is these aggregates may result from the tiny fraction of polymers with very high molecular weight that are reluctant to dissolve. The $R_{\mathrm{h}}$ of the unimer in the $\left[\mathrm{C}_{4} \mathrm{mim}\right] \mathrm{PF}_{6}$ is $<10 \mathrm{~nm}$, consistent with the size of $[\mathrm{PEG}-b-\mathrm{P}(\text { AzoMA- } r \text {-NIPAm })]_{4}$ single polymer chains with a molecular weight of $92 \mathrm{kDa}$.

When the temperature was lower than the UCMT, only an intense peak around the $R_{\mathrm{h}}$ of large particles remained and the smaller $R_{\mathrm{h}}$ peak was completely disappeared. This indicates that unimers aggregated into micelles. The $R_{\mathrm{h}}$ of the micelle at lower temperatures are approximately $35 \mathrm{~nm}$, possibly suggesting spherical core-shell micelles 

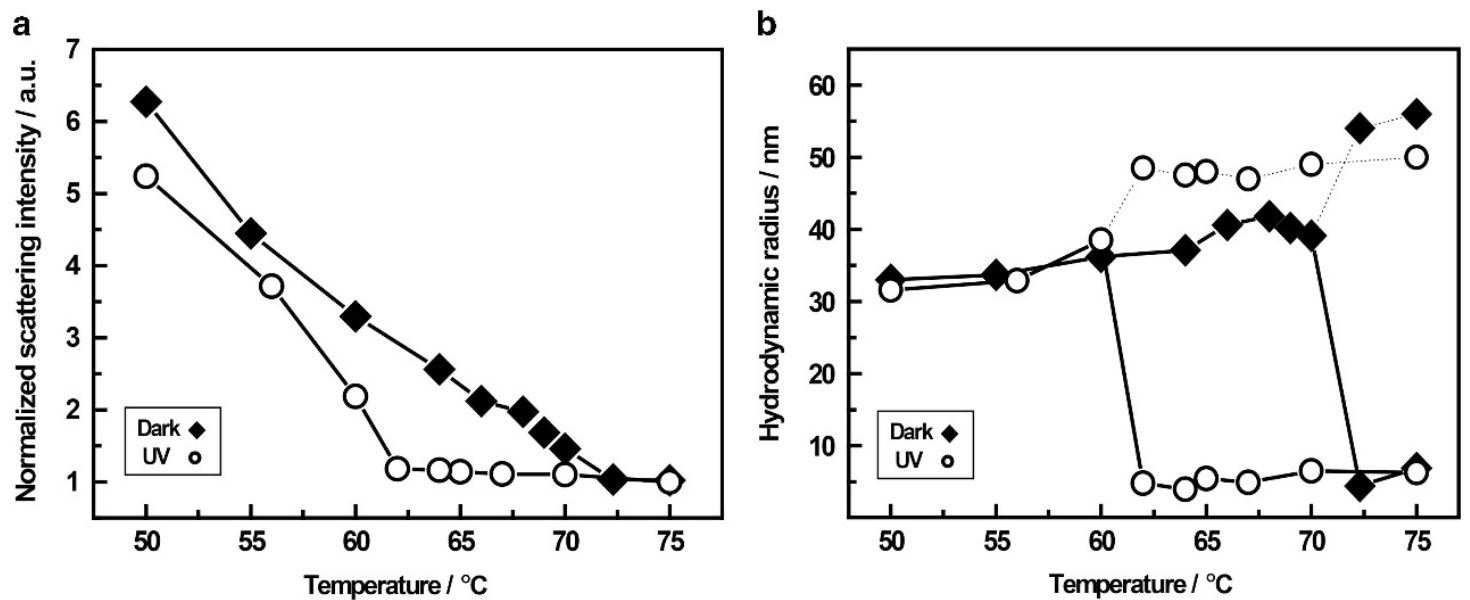

Figure 3 (a) Temperature dependence of normalized scattering intensity for [PEG- $b$-P(AzoMA-r-NIPAm) $]_{4}\left(1\right.$ wt $\%$ ) in $\left[C_{4}\right.$ mim]PF ${ }_{6}$ under dark conditions (solid diamonds) and with UV-light irradiation (open circles) at a scattering angle of $90^{\circ}$ in the cooling process. (b) Temperature dependence of the hydrodynamic radius $\left(R_{\mathrm{h}}\right)$ for $[\mathrm{PEG}-b-\mathrm{P}(\mathrm{AzoMA}-\mathrm{r}-\mathrm{NIPAm})]_{4}(1 \mathrm{wt} \%)$ in $\left[\mathrm{C}_{4} \mathrm{mim}\right] \mathrm{PF}_{6}$ under dark conditions (solid diamonds) and with UV-light irradiation (open circles). Dotted line indicates minor components in the system.

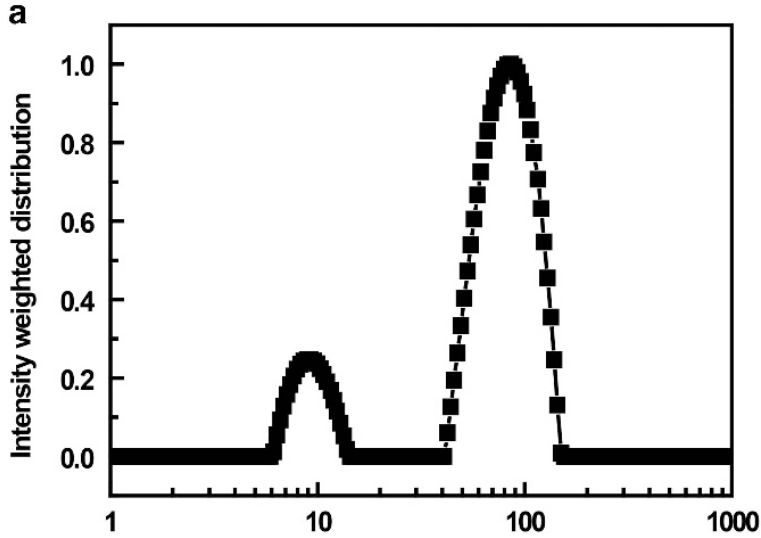

Hydrodynamic radius / $\mathrm{nm}$

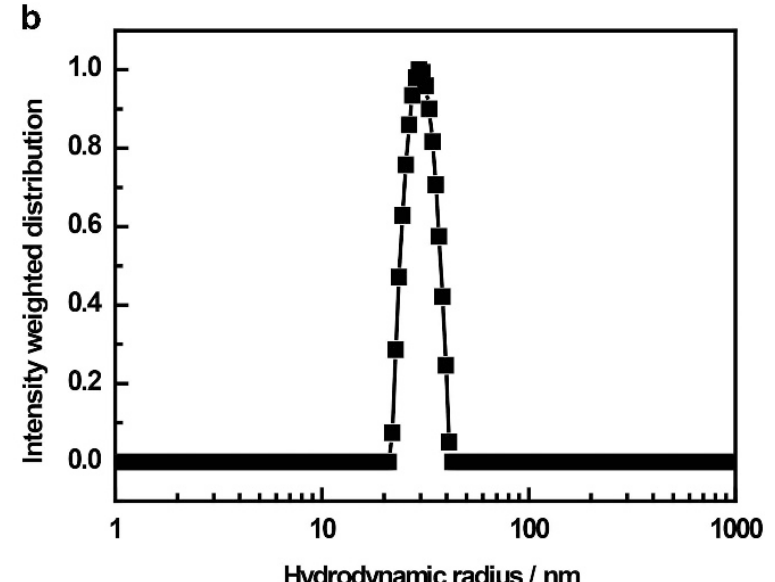

Hydrodynamic radius / $\mathrm{nm}$

Figure 4 CONTIN results for [PEG-b-P(AzoMA-r-NIPAm) $]_{4}$ in $\left[\mathrm{C}_{4}\right.$ mim]PF 6 (1 wt\%) solution at (a) $75^{\circ} \mathrm{C}$ and (b) $50^{\circ} \mathrm{C}$ under dark conditions.
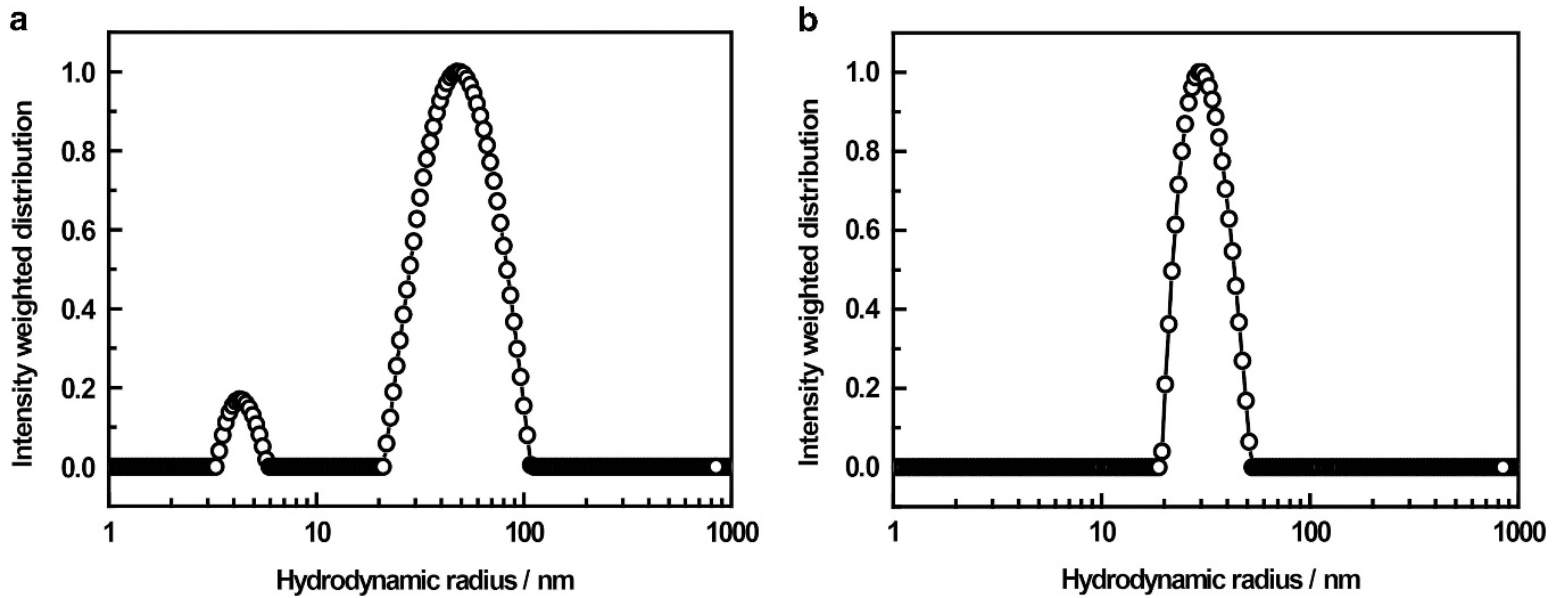

Figure 5 CONTIN results for $[\text { PEG- } b-P(A z o M A-r-N I P A m)]_{4}$ in $\left[\mathrm{C}_{4}\right.$ mim $] \mathrm{PF}_{6}(1 \mathrm{wt} \%)$ solution at (a) $75^{\circ} \mathrm{C}$ and (b) $50^{\circ} \mathrm{C}$ under UV-light irradiation. 
with solvatophobic P(AzoMA-r-NIPAm) core. The core could be surrounded by solvatophilic tetra-PEG segments that are not free endarm shells, as they have a tetra-arm nodule in the center of the block copolymer. We have previously reported thermo/photoinduced micellization of an azobenzene-containing diblock copolymer (PEO- $b$-P (AzoMA- $r$-NIPAm), total $M_{\mathrm{n}}=35 \mathrm{kDa}$, PEO weight fraction $=0.43$ ) in $\left[\mathrm{C}_{4} \mathrm{mim}\right] \mathrm{PF}_{6} \cdot{ }^{38}$ In that report, below the UCMT, PEO- $b$-P(AzoMA$r$-NIPAm) diblock copolymers self-assembled into micelles having $R_{\mathrm{h}}=120 \mathrm{~nm}$, which could have vesicular morphology judged from the $M_{\mathrm{n}} \cdot{ }^{38}$ Since the present tetra-arm block copolymer has the same PEO weight fraction of 0.43 as that of the diblock copolymer, the difference in the $R_{\mathrm{h}}$ values could mainly be attributed to a difference in the block copolymer architecture (simple diblock copolymer and tetra-arm diblock copolymer). The PEO segments in the diblock copolymer have free chain ends, whereas those in the tetra-arm block copolymer do not. Such difference appears to cause the difference in the micellar structures. After passing through the UCMT, the scattering intensity monotonically increases (Figure $3 \mathrm{a}$ ); nevertheless, the $R_{\mathrm{h}}$ of the tetraarm diblock copolymer is almost kept constant with decreasing temperature (Figure $3 \mathrm{~b}$ ). Since the scattering intensity is generally proportional to the sixth power of the size of the micelle and linear to the number of particles. Thus, this result may suggest that the number of particles gradually increases with keeping the size of micelle constant during the cooling process. ${ }^{59}$

The UCMTs of the tetra-arm diblock copolymer were 70 and $60^{\circ} \mathrm{C}$ for the trans- and cis-form polymer in $\left[\mathrm{C}_{4} \mathrm{mim}\right] \mathrm{PF}_{6}$, respectively (Figure 3b). Importantly, there is a $10^{\circ} \mathrm{C}$ difference between the UCMT of the trans-form polymer and that of the cis-form polymer (bistable temperature range). The UCMT difference arises from the difference in polarity of photoisomerization states of AzoMA. It is well-known that the polarity of azobenzene depends on its photoisomerization state; the dipole moment of the planar trans- azobenzene $(0.5 \mathrm{D})$ is markedly smaller than that of cis- azobenzene $(3.1 \mathrm{D})$. This could result in the higher polarity of the latter and its improved solubility in ILs. $\left[\mathrm{C}_{4} \operatorname{mim}\right] \mathrm{PF}_{6}$ has dipole moment $(\mu)$ of $5.3 \mathrm{D}$ and dielectric constant $(\varepsilon)$ of 11.4 , indicating moderate polarity ${ }^{60,61}$ Therefore, the trans-form polymers have higher UCMT in ILs than the cis-form polymers. In our previous report, the UCMT difference of the diblock copolymer with or without UV irradiation was as small as $4{ }^{\circ} \mathrm{C} .{ }^{38}$ The different temperature ranges between both systems can be attributed to the difference in azobenzene composition of the block copolymers. The azobenzene composition in the stimuli-responsive segment for the previously reported diblock copolymer was $2.0 \mathrm{~mol} \%$, whereas that in the present tetra-arm diblock copolymer was $8.7 \mathrm{~mol}$ $\%$. These results are consistent with the previous report on the UCST phase transition of a $\mathrm{P}$ (AzoMA-r-NIPAm) random copolymer, where the cloud point difference between the trans- and cis-form polymers changed from 4 to $24^{\circ} \mathrm{C}$ with a change in the AzoMA composition (from 1.9 to $8.6 \mathrm{~mol} \%$ ). ${ }^{38}$ By using this transition temperature difference, we further confirmed the reversibility of photoinduced unimer/micelle transition.

\section{Unimer-micelle transition of [PEG- $b$-P(AzoMA- $r$-NIPAm) $]_{4}$ in $\left[\mathrm{C}_{4} \mathrm{mim}\right] \mathrm{PF}_{6}$ induced by photo-stimuli}

Figure 6 shows a reversible unimer-micelle transition induced by light stimuli at a bistable temperature. First, the polymer solution was kept at a bistable temperature $\left(64^{\circ} \mathrm{C}\right)$ under UV-light irradiation; the $R_{\mathrm{h}}$ of the tetra-arm diblock copolymer was $<10 \mathrm{~nm}$. The size distribution always displays a bimodal distribution (Figure 7a). This is consistent with distribution function of Figure $5 \mathrm{a}$. The weight fraction of single polymer chains could also be estimated to be over 0.999 , which indicates that aggregation is negligible. At time $0 \mathrm{~s}$, the solution was irradiated by visible light to form the trans-polymer. The size of micelles formed by photoinduced transition was $\sim 35 \mathrm{~nm}$. This was quite similar to the size of the micelles generated under the thermal induced transition of [PEG- $b-\mathrm{P}($ trans-AzoMA- $r$-NIPAm $)]_{4}$, as shown in Figure $4 \mathrm{~b}$. The size distribution function exhibits a unimodal distribution (Figure $7 \mathrm{~b}$ ), which indicates formation of micelles. There is an induction time (500 s) before aggregation of the tetra-arm diblock copolymer. We previously reported that photoinduced unimer-micelle transition of PEO- $b$-P(AzoMA-r-NIPAm) diblock copolymer in an IL required two elementary steps. ${ }^{38}$ The first step was the photoisomerization of azobenzene from cis to trans, while the second step was diffusion of polymers to self-assemble into micelles. ${ }^{38}$ We considered these two steps, because the photoisomerization of the cis- to the trans-form of the azobenzene-containing random copolymer in the IL reached a photostationary state within $240 \mathrm{~s}$ under UV-light irradiation, ${ }^{20,21}$ using the same experimental set-up and conditions as the present study. Therefore, polymer diffusion step is the rate-determining step for photoinduced micellization.

After the induction time, the scattering intensity monotonically increased until $2300 \mathrm{~s}$, whilst the average $R_{\mathrm{h}}$ abruptly increased
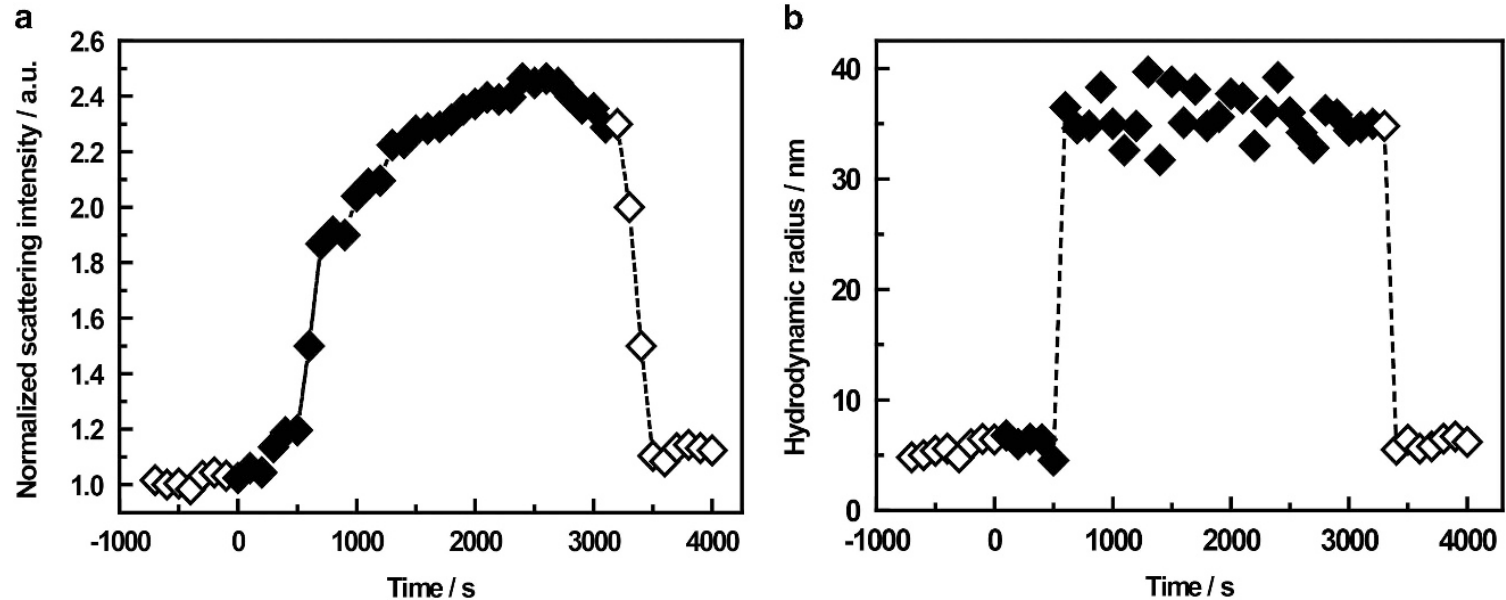

Figure 6 Reversible photoinduced self-assembly and dissociation of [PEG- $b$-P(AzoMA-r-NIPAm) $]_{4}$ polymer micelles at $64{ }^{\circ} \mathrm{C}$ under UV (open diamonds) or visible light (solid diamonds) irradiation; (a) normalized scattering intensity and (b) mean $R_{\mathrm{h}}$, as a function of time. 


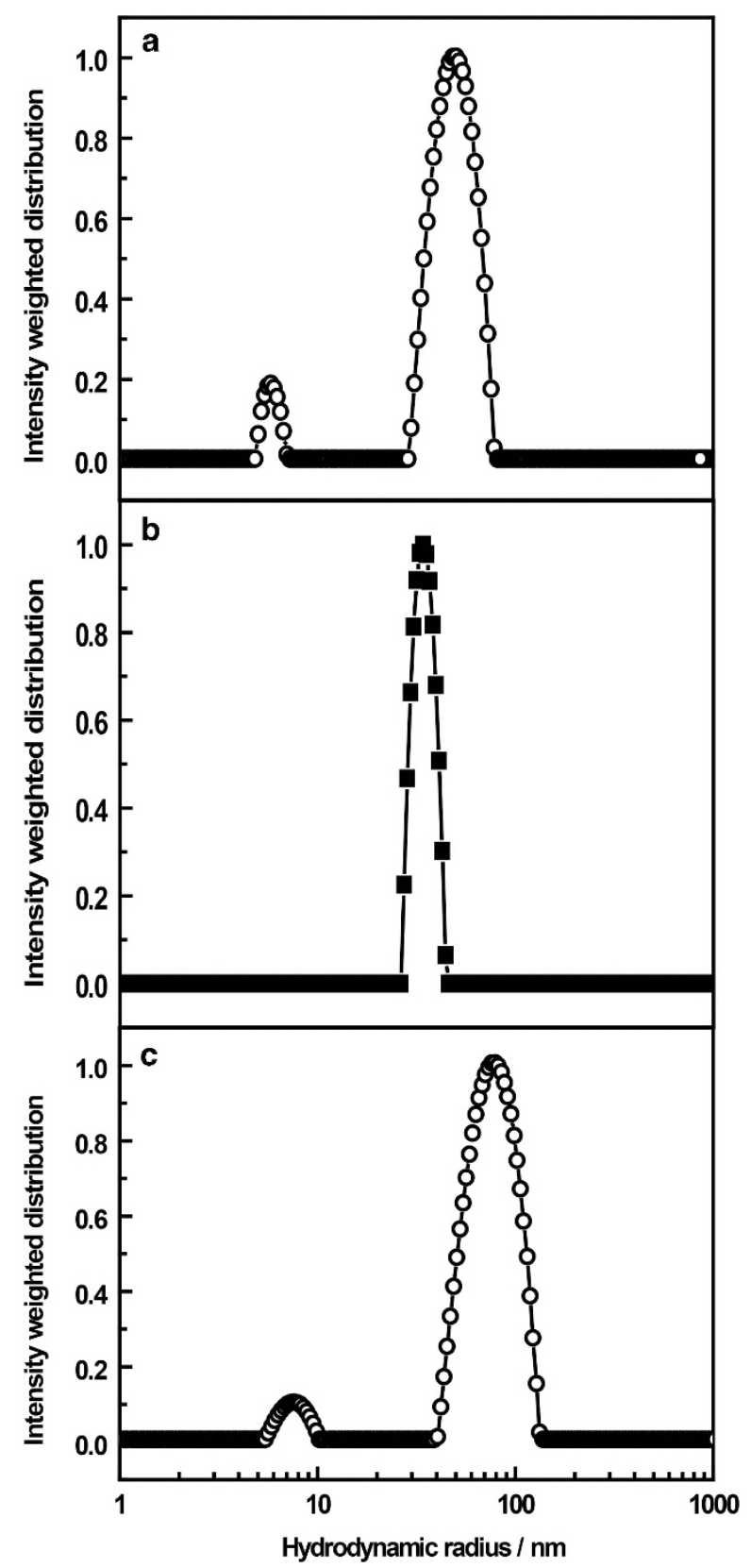

Figure 7 CONTIN results for $[\text { PEG- } b \text {-P(AzoMA-r-NIPAm) }]_{4}$ in a $\left[\mathrm{C}_{4}\right.$ mim]PF 6 (1 wt\%) solution at $64^{\circ} \mathrm{C}$ : (a) under UV-light irradiation (before visible light irradiation); (b) under visible light irradiation; and (c) after switching back to UV-light irradiation again.

(Figure 6). Early in the aggregation process, system includes a large amount of unimers and few large aggregates. The scattering intensity strongly depends on the size of particles rather than the number of particles. Therefore, once the unimers aggregation starts, the DLS signal is heavily weighted by the larger particles. ${ }^{59}$ Thus, the increase of scattering intensity from 500 to $2300 \mathrm{~s}$ is roughly proportional to the increase of the number of micelles. The completion of aggregation process seems to be reached after about $2300 \mathrm{~s}$.

After the micelles were formed under visible light irradiation, we switched back to UV-light irradiation at $3250 \mathrm{~s}$ to form the cis-form polymer. Both the scattering intensity and the $R_{\mathrm{h}}$ decreased (Figure 6). This indicated that the disassociation of micelles into individual single polymer chains, because the UCMT of the cis-form polymer was lower than $64^{\circ} \mathrm{C}$ (measurement temperature). The distribution function also confirmed this transition (Figure $7 \mathrm{c}$ ). This phenomenon demonstrated that the photoinduced unimer/micelle transition was reversible. The demicellization process was much faster than the micellization process, because no polymer diffusion step was required.

\section{CONCLUSION}

A tetra-arm diblock copolymer ([PEG- $b$-P(AzoMA- $r$-NIPAm) $\left.]_{4}\right)$ was successfully prepared by RAFT polymerization. The tetra-arm diblock copolymer had tetra-PEG as the solvatophilic central block and P (AzoMA-r-NIPAm) as four temperature- and photoresponsive end blocks. We studied its self-assembly in $\left[\mathrm{C}_{4} \mathrm{mim}\right] \mathrm{PF}_{6}$ under diluted conditions. It was shown that the UCMT of the trans-form polymer was $10^{\circ} \mathrm{C}$ higher than that of cis-form polymer from the DLS measurements. Finally, a photoinduced reversible unimer/micelle transition of the tetra-arm diblock copolymer utilizing the UCMT difference between the trans- and the cis-form polymer in an IL was demonstrated. A study on the development of photoreversible sol-gel transition using self-assembly of the tetra-arm diblock copolymers for concentrated solutions is now in progress.

\section{CONFLICT OF INTEREST}

The authors declare no competing financial interest.

\section{ACKNOWLEDGEMENTS}

This work was financially supported by the Grants-in-Aid for Scientific Research of \#A-23245046, \#S-15H05758, and the Specially Promoted Research on 'Iontronics' from the MEXT of Japan, and in part from the Research Fellowships of the Japan Society for the Promotion of Science for Young Scientists (No. 13J00192 to YK).

1 Welton, T. Room-temperature ionic liquids. solvents for synthesis and catalysis. Chem. Rev. 99, 2071-2083 (1999).

2 Holbrey, J. D. \& Seddon, K. R. Ionic liquids. Clean Products Process 1, 223-236 (1999)

3 Seddon, K. R. Ionic liquids: a taste of the future. Nat. Mater. 2, 363-365 (2003).

4 Wasserscheid, P. \& Keim, W. Ionic liquids - new "solutions" for transition metal catalysis. Angew. Chem. Int. Ed. Engl. 39, 3772-3789 (2000).

5 Magee, J. W., Kabo, G. J. \& Frenkel, M. in ACS Symposium Series 901: Ionic liquids IIIA: Fundamentals, Progress, Challenges, and Opportunities Properties and Structure (eds Rogers R. D. \& Seddon K. R.) Ch. 12, 160-174 (American Chemical Society, Washington, DC, USA, 2005).

6 Dietz, M. L., Dzielawa, J. A., Jensen, M. P., Beitz, J. V. \& Borkowski, M. in ACS Symposium Series 902: Ionic liquids IIIB: Fundamentals, Progress, Challenges, and Opportunities Transformations and Processed (eds Rogers R. D. \& Seddon K. R.) Ch. 1, 2-18 (American Chemical Society, Washington, DC, USA, 2005).

7 Wilkes, J. S. A short history of ionic liquids-from molten salts to neoteric solvents. Green Chem. 4, 73-80 (2002).

8 Plechkova, N. V. \& Seddon, K. R. Applications of ionic liquids in the chemical industry. Chem. Soc. Rev. 37, 123-150 (2008).

9 Holbrey, J. D., Chen, J., Turner, M. B., Swatloski, R. P., Spear, S. K. \& Rogers, R. D. in ACS Symposium Series 913: lonic liquids in Polymer Systems: Solvents, Additives, and Novel Application (eds Brazel C. S. \& Rogers R. D.) Ch. 5, 71-87 (American Chemical Society, Washington, DC, USA, 2005).

10 Winterton, N. Solubilization of polymers by ionic liquids. J. Mater. Chem. 16, 4281-4293 (2006).

11 Lodge, T. P. Materials science: a unique platform for materials design. Science $\mathbf{3 2 1}$, 50-51 (2008).

12 Ueki, T. \& Watanabe, M. Polymers in ionic liquids: dawn of neoteric solvents and innovative materials. Bull. Chem. Soc. Jpn. 85, 33-50 (2012).

13 Ueki, T. \& Watanabe, M. Upper critical solution temperature behavior of poly( $\mathrm{N}$ isopropylacrylamide) in an ionic liquid and preparation of thermo-sensitive nonvolatile gels. Chem. Lett. 35, 964-965 (2006).

14 Ueki, T. \& Watanabe, M. Lower critical solution temperature behavior of linear polymers in ionic liquids and the corresponding volume phase transition of polymer gels. Langmuir 23, 988-990 (2007). 
15 Kodama, K., Nanashima, H., Ueki, T., Kokubo, H. \& Watanabe, M. Lower critical solution temperature phase behavior of linear polymers in imidazolium-based ionic liquids: effects of structural modifications. Langmuir 25, 3820-3824 (2009).

16 Lee, H. -N. \& Lodge, T. P. Poly ( $n$-butyl methacrylate) in ionic liquids with tunable lower critical solution temperatures (LCST). J. Phys. Chem. B 115, 1971-1977 (2011).

17 Kodama, K., Tsuda, R., Niitsuma, K., Tamura, T., Ueki, T., Kokubo, H. \& Watanabe, M. Structural effects of polyethers and ionic liquids in their binary mixtures on lower critical solution temperature liquid-liquid phase separation. Polym. J. 43, 242-248 (2011).

18 Lee, H.-N., Newell, N., Bai, Z. \& Lodge, T. P. Unusual lower critical solution temperature phase behavior of poly(ethylene oxide) in ionic liquids. Macromolecules 45, 3627-3633 (2012)

19 Rodriguez, H. \& Rogers, R. D. Liquid mixtures of ionic liquids and polymers as solvent systems. Fluid Phase Equilibria 294, 7-14 (2010).

20 Ueki, T., Nakamura, Y., Yamaguchi, A., Niitsuma, K., Lodge, T. P. \& Watanabe, M. UCST phase transition of azobenzene-containing random copolymer in an ionic liquid. Macromolecules 44, 6908-6914 (2011).

21 Ueki, T., Yamaguchi, A., Ito, N., Kodama, K., Sakamoto, J., Ueno, K., Kokubo, H. \& Watanabe, M. Photoisomerization-induced tunable LCST phase separation of azobenzenecontaining polymers in an ionic liquid. Langmuir 25, 8845-8848 (2009).

22 Riess, G. Micellization of block copolymers. Prog. Polym. Sci. 28, 1107-1170 (2003).

23 Hamley, I. W. Block Copolymers in Solutions: Fundamentals and Applications, (John Wiley \& Sons, Chichester, UK, 2005)

24 Alexandridis, P. \& Lindman, B. in Amphiphilic Block Copolymers: Self-Assembly and Applications (eds Alexandridis, P. \& Lindman, B.) Ch. 1, 1-12 (Elsevier, Amsterdam, The Netherlands, 2000).

25 Zhang, L. \& Eisenberg, A. Multiple morphologies of "crew-cut" aggregates of polystyrene- $b$-poly(acrylic acid) block copolymers. Science 268, 1728-1731 (1995).

26 Bates, F. S. Polymer-polymer phase behavior. Science 251, 898-905 (1991).

$27 \mathrm{He}$, Y., Li, Z., Simone, P. \& Lodge, T. P. Self-assembly of block copolymer micelles in an ionic liquid. J. Am. Chem. Soc. 128, 2745-2750 (2006).

$28 \mathrm{He}$, Y., Boswell, P. G., Buhlmann, P. \& Lodge, T. P. Ion gels by self-assembly of a triblock copolymer in an ionic liquid. J. Phys. Chem. B 111, 4645-4652 (2007).

29 Cho, J. -H., Lee, J., Xia, Y., Kim, B. -S., He, Y., Renn, M. J., Lodge, T. P. \& Frisbie, C. D. Printable ion-gel gate dielectrics for low-voltage polymer thin-film transistors on plastic. Nat. Mater. 7, 900-906 (2008).

30 Imaizumi, S., Kokubo, H. \& Watanabe, M. Polymer actuators using ion-gel electrolytes prepared by self-assembly of ABA-triblock copolymers. Macromolecules 45 401-409 (2012)

31 Moon, H. C., Lodge, T. P. \& Frisbie C. D. Solution-processable electrochemiluminescent ion gels for flexible, low-voltage, emissive displays on plastic. J. Am. Chem. Soc. 136 3705-3712 (2014)

32 Tamura, S., Ueki, T., Ueno, K., Kodama, K. \& Watanabe, M. Thermosensitive selfassembly of diblock copolymers with lower critical micellization temperatures in ionic liquid. Macromolecules 42, 6239-6244 (2009).

33 Ueki, T., Watanabe, M. \& Lodge, T. P. Doubly thermosensitive self-assembly of diblock copolymers in ionic liquids. Macromolecules 42, 1315-1320 (2009).

34 Lee, H. -N., Bai, Z., Newell, N. \& Lodge, T. P. Micelle/inverse micelle self-assembly of a PEO-PNIPAm block copolymer in ionic liquids with double thermoresponsivity. Macromolecules 43, 9522-9528 (2010).

$35 \mathrm{He}$, Y. \& Lodge, T. P. A thermoreversible ion gel by triblock copolymer self-assembly in an ionic liquid. Chem. Commun. 43, 2732-2734 (2007).

$36 \mathrm{He}$, Y. \& Lodge, T. P. Thermoreversible ion gels with tunable melting temperatures from triblock and pentablock copolymers. Macromolecules 41, 167-174 (2008).

37 Kitazawa, Y., Ueki, T., Niitsuma, K., Imaizumi, S., Lodge, T. P. \& Watanabe, M. Thermoreversible high-temperature gelation of an ionic liquid with poly(benzyl methacrylate-b-methyl methacrylate- $b$-benzyl methacrylate) triblock copolymer. Soft Matter $\mathbf{8}$ 8067-8074 (2012).

38 Ueki, T., Nakamura, Y., Lodge, T. P. \& Watanabe, M. Light-controlled reversible micellization of a diblock copolymer in an ionic liquid. Macromolecules 45 , 7566-7573 (2012).

39 Ueki, T., Nakamura, Y., Usui, R., Kitazawa, Y., So, S., Lodge, T. P. \& Watanabe, M. Photoreversible gelation of a triblock copolymer in an ionic liquid. Angew. Chem. Int. Ed. Engl. 54, 3018-3022 (2015).
40 Strandman, S., Zarembo, A., Darinskii, A. A., Loflund, B., Butcher, S. J. \& Tenhu, H. Self-assembling of star-like amphiphilic block copolymers with polyelectrolyte blocks. Effect of pH. Polymer 48, 7008-7016 (2007).

$41 \mathrm{He}$, E., Ravi, P. \& Tam, K. C. Synthesis and self-assembly behavior of four-arm poly (ethylene oxide)-b-poly(2-(diethylamino)ethyl methacrylate) star block copolymer in salt solutions. Langmuir 23, 2382-2388 (2007).

42 Mori, H., Ebina, Y., Kambara, R. \& Nakabayashi, K. Temperature-responsive selfassembly of star block copolymers with poly(ionic liquid) segments. Polymer J 44, 550-560 (2012).

43 Jeong, Y., Nah, J., Lee, H., Kim, S. \& Cho, C. Adriamycin release from flower-type polymeric micelle based on star-block copolymer composed of poly $(\gamma$-benzyl L-glutamate) as the hydrophobic part and poly(ethylene oxide) as the hydrophilic part. Int. J. Pharm. 188, 49-58 (1999).

$44 \mathrm{He}$, E., Yue, C. Y. \& Tam, K. C. Association behavior of star-shaped ph-responsive block copolymer: four-arm poly(ethylene oxide)-b-poly(methacrylic acid) in aqueous medium. Langmuir 25, 4892-4899 (2009).

45 Deng, M., Chen, X., Piao, L., Zhang, X., Dai, Z. \& Jing, X. Synthesis of four-armed poly ( $\varepsilon$-caprolactone)-block-poly(ethylene oxide) by diethylzinc catalyst. J. Polym. Sci. A Polym. Chem 4, 950-959 (2004).

46 Kim, K., Cui, G., Lim, H., Huh, J., Ahn, C. H. \& Jo, W. Synthesis and micellization of star-shaped poly(ethylene glycol)-block-poly( $\varepsilon$-caprolactone). Macromol. Chem. Phys. 205, 1684-1692 (2004).

47 Jones, M. C., Ranger, M. \& Leroux, J. C. pH-Sensitive unimolecular polymeric micelles: synthesis of a novel drug carrier. Bioconjug. Chem. 14, 774-781 (2003).

48 Ryayev, J. \& Hillmyer, M. A. Nanochannel array plastics with tailored surface chemistry. J. Am. Chem. Soc. 127, 13373-13379 (2005).

49 Lai, J. T., Filla, D. \& Shea, R. Functional polymers from novel carboxyl-terminated trithiocarbonates as highly efficient RAFT agents. Macromolecules 35, 6754-6756 (2002).

50 Yuan, W., Jiang, G., Wang, J., Wang, G., Song, Y. \& Jiang, L. Temperature/light dualresponsive surface with tunable wettability created by modification with an azobenzenecontaining copolymer. Macromolecules 39, 1300-1303 (2006).

51 Shimoboji, T., Ding, Z., Stayton, P. \& Hoffman, A. Photoswitching of ligand association with a photoresponsive polymer-protein conjugate. Bioconjug. Chem. 13, 915-919 (2002).

52 Tokuda, H., Hayamizu, K., Ishii, K., Susan, M. A. \& Watanabe, M. Physicochemical properties and structures of room temperature ionic liquids. 1. Variation of anionic species. J. Phys. Chem. B 108, 16593-16600 (2004).

53 Brown, W. Dynamic Light Scattering: The Method and Some Applications, (Clarendon Press, Oxford, UK, 1993)

54 Koppel, D. E. Analysis of macromolecular polydispersity in intensity correlation spectroscopy. Method of cumulants. J. Chem. Phys. 57, 4814-4820 (1972).

55 Provencher, S. W. A constrained regularization method for inverting data represented by linear algebraic or integral equations. Comput. Phys. Commun. 27 213-227 (1982)

56 Pereiro, A. B., Legido, J. L. \& Rodríguez, A. Physical properties of ionic liquids based on 1-alkyl-3-methylimidazolium cation and hexafluorophosphate as anion and temperature dependence. J. Chem. Thermodynamics 39, 1168-1175 (2007).

57 Luo, C., Zuo, F., Ding, X., Zheng, Z., Cheng, X. \& Peng, Y. Light-triggered reversible solubility of $\alpha$-cyclodextrin and azobenzene moiety complexes in PDMAA-co-PAPA via molecular recognition. J. Appl. Polym. Sci. 4, 2118-2125 (2008).

58 Ueki, T., Yamaguchi, A. \& Watanabe, M. Unlocking of interlocked heteropolymer gel by light: photoinduced volume phase transition in an ionic liquid from a metastable state to an equilibrium phase. Chem. Commun. 48, 5133-5135 (2012).

59 Shibayama, M., Karino, T. \& Okabe, S. Distribution analyses of multi-modal dynamic light scattering data. Polymer 47, 6446-6456 (2006).

60 Hunger, J., Stoppa, A., Schrodle, S., Hefter, G. \& Buchner, R. Temperature dependence of the dielectric properties and dynamics of ionic liquids. ChemPhysChem 10 723-733 (2009).

61 Wakai, C., Oleinikikova, A., Ott, M. \& Weingartner, H. How polar are ionic liquids? determination of the static dielectric constant of an imidazolium-based ionic liquid by microwave dielectric spectroscopy. J. Phys. Chem. B 109, 17028-17030 (2005).

Supplementary Information accompanies the paper on Polymer Journal website (http://www.nature.com/pj) 